\title{
Conférence internationale sur les femmes en physique
}

Organisée par le Working Group 5 ('Women in Physics') de l'IUPAP (International Union of Pure and Applied Physics), à Birmingham, UK, en juillet 2017

Compte-rendu de Véronique Pierron-Bohnes (vero@ipcms.unistra.fr) et Nathalie Westbrook

pour la Société Française de Physique (SFP), I'association Femmes et Sciences et la Société Française d'Optique (SFO)

La sixième Conférence internationale sur les femmes en physique (ICWIP 2017) a eu lieu à Birmingham, du 16 au 20 juillet 2017. Cette conférence a réuni 210 personnes de 47 pays et a proposé six conférences plénières représentant des disciplines et des pays variés, deux séances posters (posters nationaux sur les femmes en physique dans les différents pays et posters scientifiques), cinq ateliers ainsi que deux évènements exceptionnels.

\section{Conférences plénières}

\section{Gender Balance in Science: an astronomer's view}

Maria Teresa Lago, Université de Porto, Portugal

Professeure émérite d'astronomie à l'Université de Porto. Recherche en astrophysique stellaire. En 1988, elle fonde l'Astrophysics Research Center de Porto qu'elle dirigera pendant 18 ans. Membre fondatrice de l'European Astronomical Society (1990), d'EuroScience (1997) et de l'European Research Council (2005). Titulaire de la chaire de l'ERC Working Group on Gender Balance entre 2007 et 2013.

Elle nous a décrit les sciences et l'astronomie au Portugal, puis comment elle a instauré dans son institut la règle que toute personne doit consacrer $10 \%$ de son temps de travail à des travaux d'intérêt collectif. Cela permet de rétablir l'équilibre pour les femmes, souvent volontaires un peu forcées pour ces tâches collectives. Maria Lago défend systématiquement à l'ERC ou pour les autres financements les personnes qui sont à temps partiel, car on ne pénalise pas ceux qui mènent dix projets en parallèle sous prétexte qu'ils sont à temps partiel sur chacun d'eux. Elle nous a donné quelques chiffres sur la proportion de femmes aux bourses de l'ERC (éligibles/demandeurs/financés) : Starting grants (30\% / $31 \% / 27 \%)$, Advanced grants (25\% / 15\% / 14\%).

\section{Blowing hot and cold in quantum technologies}

Halina Rubinsztein-Dunlop, Université de Queensland, Australie

Études à l'Université de Technologie de Chalmers, à Göteborg en Suède. Directrice du Quantum Science Laboratory de Queensland, où elle anime un important groupe de recherche en optique atomique expérimentale, micromanipulation laser et nano-optique. En 2003, elle a rę̧u le prix
AIP Women in Physics Lecturer de l'Institut australien de physique. Son groupe a publié plus de 225 articles scientifiques internationaux de fort impact.

Elle a décrit les résultats de ses recherches sur les condensats de Bose-Einstein, qui offrent la possibilité unique de faire des mesures de thermodynamique quantique sur des systèmes à $\mathrm{n}$ corps totalement isolés. Son groupe a développé des méthodes pour "sculpter " un faisceau laser afin de créer des potentiels de configuration choisie avec une résolution en dessous du micron. Par exemple, la seconde loi de la thermodynamique a ainsi pu être étudiée à température non nulle à l'échelle atomique.

\section{Magnetospheric Physics}

Francisca Nneka Okeke, Africa Climate Change Adaptation Initiative, Université du Nigéria

Professeure de physique, ses recherches portent sur la géophysique de la Terre solide, la physique atmosphérique et les changements climatiques. Elle a obtenu le prix L'Oréal-UNESCO pour les Femmes et la Science en 2013. Elle a écrit des livres et des articles de base en physique qui ont permis d'augmenter le niveau de l'enseignement et des études de la physique au Nigéria, ainsi que de nombreux articles pour encourager les filles et les femmes à s'intéresser aux sciences en Afrique.

Elle nous a présenté une vue d'ensemble des magnétosphères des différentes planètes du système solaire et de leurs interactions avec les particules qui arrivent du Soleil ou de l'espace. Son travail a aidé à comprendre les effets des courants ioniques en haute atmosphère sur le climat des pays équatoriaux. Elle a aussi décrit son parcours scientifique au Nigéria. 


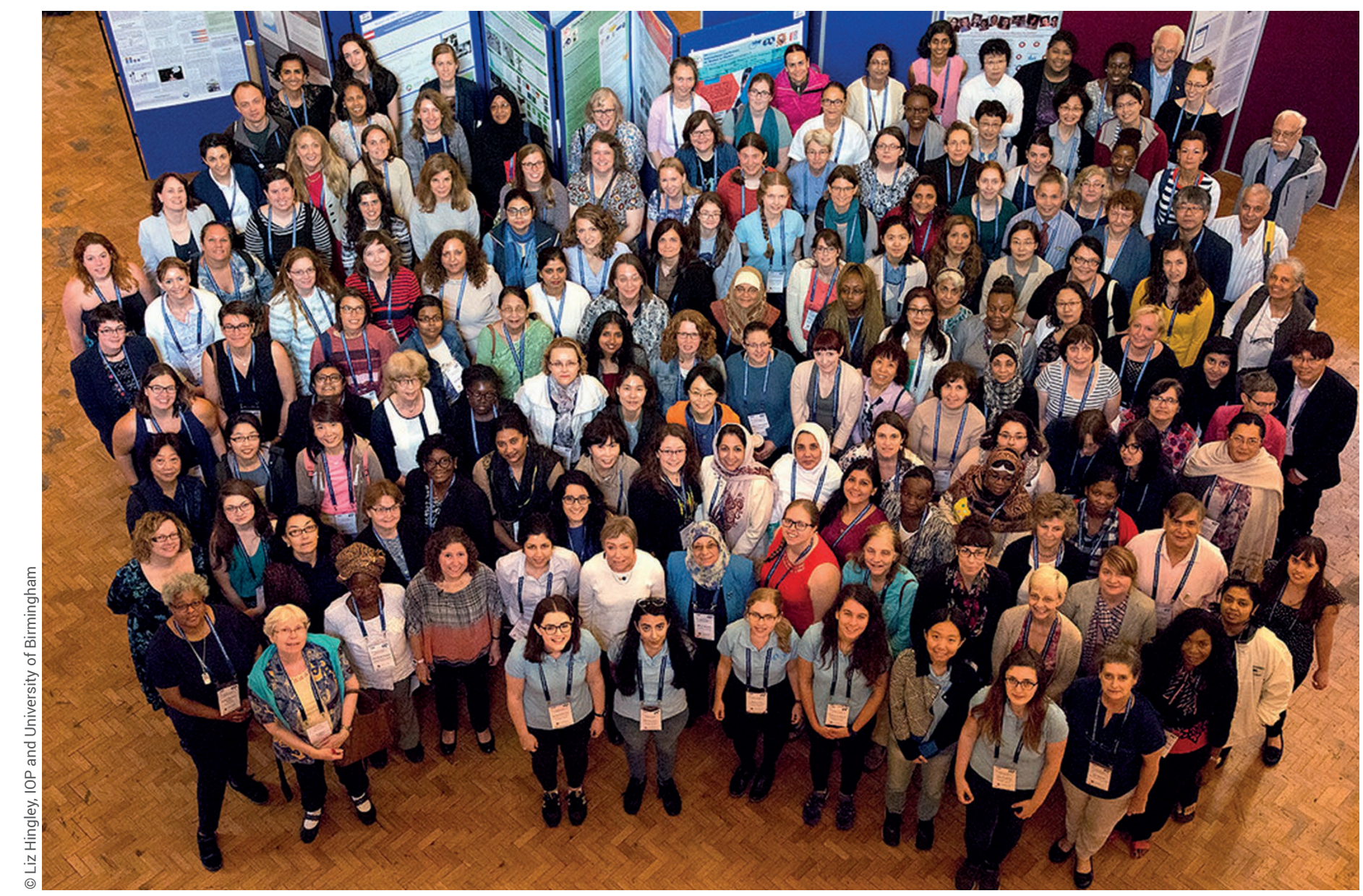

Les participantes de la conférence.

\section{Quantum Effects in Nanostructures}

Xucun Ma, Université Tsinghua, Chine

Professeure de physique, ses principaux sujets de recherche sont la croissance des films minces et les effets $d u$ confinement quantique dans les matériaux nanostructurés. La mise en évidence par son groupe de la supraconductivité à haute température augmentée par l'interface dans les hétérostructures $\mathrm{FeSe} / \mathrm{SrTiO} \mathrm{T}_{3}\left(\mathrm{~T}_{c}>77 \mathrm{~K}\right)$ a été considérée comme la découverte la plus importante des cinq dernières années dans le domaine des supraconducteurs à haute $T_{c}$.

Elle a essentiellement parlé de sa recherche : le rôle de la surface et du substrat dans la supraconductivité de ces composés et dans les propriétés des isolants topologiques. Elle n'a pas ressenti de problème comme femme scientifique en Chine et affirme que le succès vient par le travail individuel, mais aussi en équipe. " $A$ good scientist must be a diligent and plucky person, always willing to exchange ideas and good at cooperating with others."

\section{Searching for - and finding! - gravitational waves}

Gabriela González, Louisiana State University, USA

Gabriela González est professeure de physique et d'astronomie à l'Université d'État de Louisiane, et a été porte-parole de la collaboration scientifique LIGO pour l'observation expérimentale des ondes gravitationnelles.
Elle nous a décrit sa démarche de plus de trente ans qui lui a permis de contribuer à la découverte des ondes gravitationnelles. Ce travail d'équipe commence par la prédiction, se prolonge par beaucoup d'instrumentation, puis par l'observation extraite du bruit grâce à la coïncidence entre les résultats des deux installations LIGO.

\section{Reflections on Not Fitting in}

Prof. Dame Athene Donald, Université de Cambridge, UK Études et majorité de sa carrière de recherche à l'Université de Cambridge, à l'interface entre la physique de la matière molle et la biologie. "Gender Equality Champion" pour l'Université de Cambridge, lauréate 2009 du prix L'Oréal-UNESCO et membre de nombreux comités nationaux et internationaux, dont le conseil scientifique de l'ERC.

Elle a présenté son parcours et ses recherches aux frontières entre la physique des polymères, la chimie et la biologie, qui ont été parmi les premières présentant une telle interdisciplinarité. Elle a développé de nouvelles techniques de microscopie électronique et de diffraction des rayons $\mathrm{X}$ adaptées à ce domaine. Elle a aussi exposé la difficulté (et les avantages) de travailler dans un secteur neuf, encore inexploré, mais avec très peu d'audience. 


\section{Séances posters}

Elles ont combiné des posters nationaux et des posters de recherche des participantes. Chaque pays a montré sur son affiche soit des statistiques actuelles dans son pays, soit une action phare. Sur le poster français (photo ci-contre), la charte gender fairness at conferences $^{(\mathrm{a})}$ a été décrite.

Nous avons à plusieurs reprises présenté la charte de la Société Française de Physique pour la parité aux conférences. Cette charte a par la suite été envoyée par courriel aux country team leaders en expliquant la démarche attendue : adapter l'introduction et les statistiques à leur pays, contacter la société nationale de physique et les entités concernées pour leur demander d'envoyer la charte à tous les organisateurs de conférences en conditionnant leur aide à sa signature, récolter les rapports de fin de conférence et alerter l'entité aidante en cas de non-respect criant. Nous avons reçu un très bon accueil de la part de nombreux pays et la charte apparait dans les recommandations du congrès à l'IUPAP.

Certains pays (Pays-Bas, Argentine) ont déjà une action équivalente. D'autres sont en train de mettre en place de telles règles (Royaume-Uni, USA, Allemagne). Les représentantes de plusieurs pays vont essayer de promouvoir l'action (au Canada, la commission WIP a déjà proposé une telle charte ; au Portugal, en Irlande, en

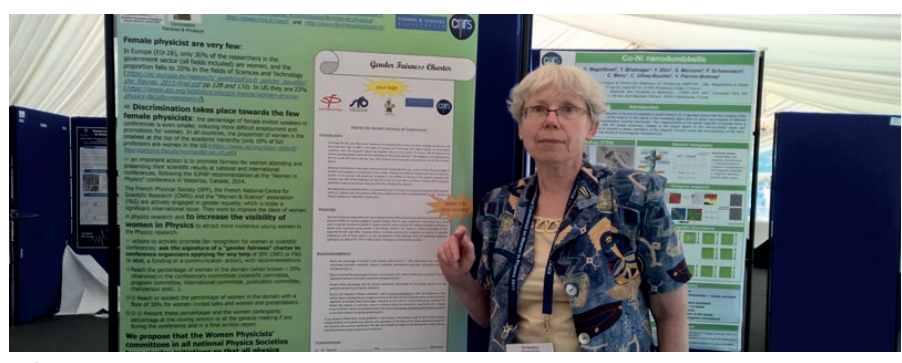

Véronique Pierron-Bohnes devant le poster français.

Finlande, en Lituanie, au Mexique, au Japon et à Taiwan, les team leaders vont la proposer ; en Inde, en Tunisie et au Cameroun, elles vont faire de leur mieux, mais sans grand espoir). Pour d'autres pays comme le Pakistan, les femmes n'ont pas ce type de préoccupations, car elles sont encore aux prises avec des problèmes de scolarisation ou de nombre extrêmement réduit de femmes en physique (et plus généralement en sciences de l'ingénieur et en mathématiques).

Les membres présentes du groupe de travail WG5 ont demandé au conseil exécutif de l'IUPAP que les recommandations qui se trouvent sur son site ${ }^{(b)}$ soient vraiment appliquées.

\section{Ateliers}

\section{A. Science et éducation}

Plusieurs points saillants sont apparus lors des discussions :

- inclure les parents et les familles dans les actions,

- prévoir des interventions récurrentes plutôt que des actions isolées,

- permettre des réflexions régulières sur ce qui est appris : pourquoi est-ce important ? où cela intervient-il dans ma vie ?

- donner aux enseignants de primaire qui n'ont pas de bagage scientifique une formation sur la démarche scientifique,

- organiser des activités où les élèves/étudiants sont actifs, avec un aspect ludique, les adapter aux différents groupes d'âge et utiliser du matériel de la vie de tous les jours,

- attirer les filles qui aiment l'art, les couleurs, la mode, etc., vers la physique en soignant l'esthétique lors des expériences,

- encourager les garçons à se joindre aux activités liant la physique et l'art, pour contrer les stéréotypes auxquels ils sont soumis,

- veiller à ce que la physique reste synonyme de modernité, avec des activités qui suivent les tendances et les technologies actuelles.

\section{B. Pratique de la science et éthique}

Tony Mayer (Nanyang Technological University, Singapour) a présenté un exposé sur l'éthique en recherche. Jenny Dyer (Head of Diversity, IOP) et Val Gibson (Cavendish Laboratory, Cambridge) ont décrit les programmes Juno et Athena SWAN de l'Institute of Physics (IOP) britannique.

L'ébauche de la charte pour les femmes en physique de Waterloo $^{(c)}$ a été peaufinée avant sa présentation au prochain comité exécutif de l'IUPAP.

\section{Perception culturelle et préjugés}

Une première session a traité des préjugés implicites et de leur prise de conscience.

- La plupart des femmes de couleur font face à des discriminations et des micro-agressions.

- Comment induire des changements culturels ? (Donner aux personnes les moyens de lutter contre les impacts des préjugés implicites, des stéréotypes, etc.)

- Étude de cas : situations vécues en tant que victime, amie ou collègue, conseiller, responsable administratif...

- Lutter contre les préjugés implicites peut améliorer l'environnement de la discipline : il faut que les plus grands défis rencontrés par les femmes soient ceux posés par la physique et non par leur environnement.

Une deuxième session a été consacrée aux actions de l'IOP pour améliorer l'équilibre de genre en physique au lycée :

- faire des actions au niveau de toute l'école,

- développer un cadre pour signaler et gérer les cas de harcèlement sexuel et moral de tous les groupes sous-représentés,

- encourager et féliciter les hommes qui encouragent les femmes en physique,

- décrire aux élèves la vie de femmes qui ont eu des succès en physique pour qu'elles puissent leur servir de modèles.

Une troisième session a porté sur la diversité culturelle et les projets multinationaux (par exemple, les projets Shemera et Target) qui ont un rôle important dans les problèmes de genre. Il faut cependant que les participants prennent en compte les spécificités nationales et soient attentifs aux préjugés inconscients. 


\section{Développement professionnel et prise de responsabilité}

La première session a commencé par une présentation de Claudia Eberlein, physicienne d'origine allemande (de l'Est) qui a fait l'essentiel de sa carrière en Angleterre et est maintenant Head of Department of Physics and Astronomy à l'Université du Sussex. Elle a présenté son parcours et les difficultés rencontrées pour atteindre son poste de full professor et sa responsabilité de département.

La seconde session visait à discuter ce qui, lors de ses études secondaires, voire dans son éducation, peut amener une fille à choisir la physique. Daniela Bortoletto (Université d'Oxford) a présenté les conférences CUWIP, qui regroupent les filles qui font des études de niveau undergraduate en physique (USA et UK), ainsi que les groupes de discussion Women in physics (aux niveaux undergraduate et graduate), pour motiver les filles à continuer. Florence Mutonyi D'ujanga (Université de Makerere, Ouganda) a présenté ses actions de sensibilisation des jeunes femmes ougandaises à la physique. Le soutien des parents ou de l'entourage, la volonté d'indépendance sont des facteurs importants. Un autre point clé consiste à parler de la sous-représentation des femmes en physique, car les jeunes femmes comprendront ainsi que le problème vient de la société et non d'elles-mêmes. Cela les aidera à se forger une identité en tant que physiciennes. Les premières femmes responsables de département peuvent encourager d'autres femmes par leur exemple, et servir de modèle en retournant dans leur village pour montrer qu'on peut réussir en étant physicienne. Les efforts des gouvernements sont également très importants.

Lors de la troisième session, un physicien de Tä̈wan, Minn-Tsong Lin, a montré comment un changement du mode d'évaluation des projets de recherche a conduit à une augmentation du taux de succès parmi les femmes scientifiques. Plus précisément, il s'agissait d'éviter les jugements purement quantitatifs, de prendre en compte la contribution des différents coauteurs, et de faire en sorte que les évaluateurs prennent le temps de contacter l'entourage académique des candidats.

L'atelier s'est terminé par une discussion sur les recommandations à faire ressortir lors du bilan. La charte proposée par la France a été discutée à ce moment-là comme recommandation à faire, car plusieurs participants l'avaient remarquée lors de la session poster.

\section{Évènements sociétaux}

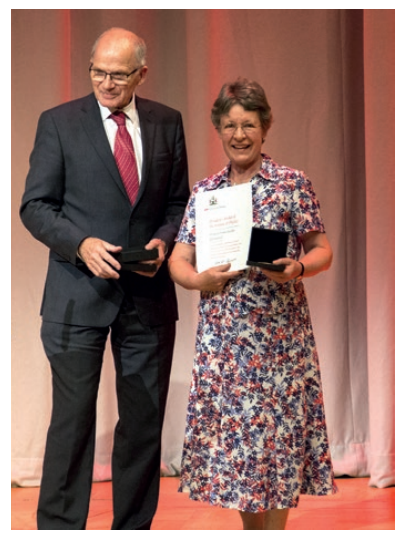

Roy Sambles

et Jocelyn Bell Burnell
1) Remise de la Médaille du Président de l'IOP à Prof. Dame Jocelyn Bell Burnell, "for her outstanding contributions to physics through pioneering research in astronomy, most notably the discovery of the first pulsars, and through her unparalleled record of leadership within the community", par le président actuel de l'IOP, Prof. Roy Sambles.

C'est grâce à sa persévérance que les objets exotiques et inattendus que sont les pulsars ont été découverts pour la première fois. Ils sont devenus des outils puissants pour explorer la physique de l'extrême et tester la relativité générale.

Jocelyn Bell Burnell occupe aujourd'hui de grandes responsabilités au sein des communautés de la physique et de l'astronomie : présidente de la Royal Astronomical Society et de l'Institute of Physics, entre autres. Elle a participé à la création du prix Athena SWAN qui récompense celles et ceux qui s'engagent pour faire progresser les carrières des femmes en sciences. En 2016, le prix 'Very Early Career Female Physicist Award', décerné par l'IOP Women in Physics Group, est devenu le prix 'Jocelyn Bell Burnell' en son honneur. Elle a été primée par des prix de plus de 35 institutions.
2) Visite surprise de Malala Yousafzai ${ }^{(d)}$, prix Nobel de la paix en 2014 pour son action sur les réseaux sociaux dès l'âge de onze ans, en faveur de la scolarisation des femmes au Pakistan et dans les pays en voie de développement.

Après avoir été blessée par les talibans en 2013, elle a été soignée à Cambridge où elle vient de finir ses études secondaires. Elle va commencer des études "Politics, Philosophy and Economics" à Oxford.

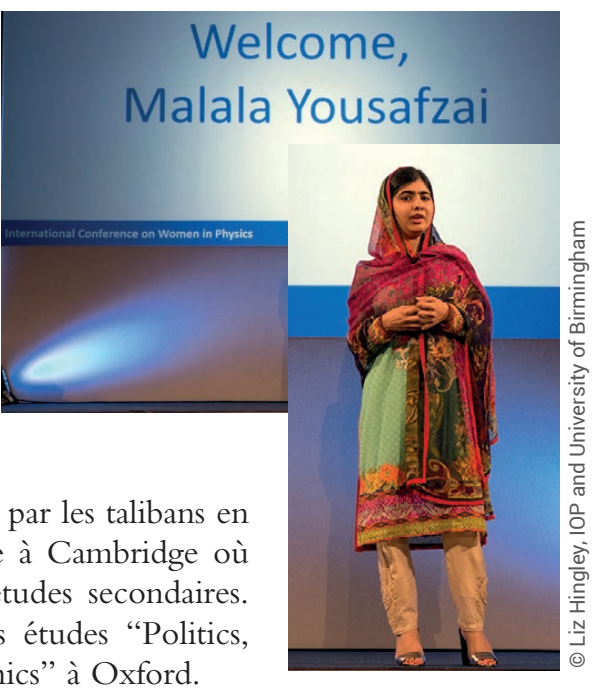

Malala a raconté ses actions dans les pays où les jeunes filles n'ont pas le droit à une scolarité sereine. Elle a décrit sa fondation qui construit des écoles, et ses voyages pour soutenir la scolarisation des filles, notamment au Liban pour les réfugiées syriennes, en Jordanie, au Nigéria, à Gaza. Elle a beaucoup ému les participantes des pays où la scolarisation des femmes reste problématique, et impressionné les autres par ses propos responsables et d'une grande sagesse pour ses vingt ans. "I decided to speak out because there was no other option. If you stay silent, nothing will change."

(a) www.sfpnet.fr/charte-de-parite-pour-les-conferences-scientifiques, charte signée par la SFP, la SFO et l'INP du CNRS.

(b) http://iupap.org/sponsores-conferences/conference-policies/ , paragraphe Women in physics: "The presence of women on the local organising committee, the international advisory committee and as plenary and invited speakers is a condition for IUPAP sponsorship. The application should include a list of the organising and advisory committees as evidence that women are included, and the numbers of women invited speakers should be included in the application and updated when the approved financial support for the sponsored conference is requested. The conference report will include the number of women speakers and attendees at the conference."

(c) http://wgwip.df.uba.ar/Waterloo Charter_Ver5p.pdf

(d) Des mesures de sécurité spéciales ont été prises (secret jusqu'à quelques minutes avant son arrivée ;interdiction de faire des photos et d'envoyer des sms qui en parlent jusqu'à ce qu'elle soit partie). Seules les représentantes de certains pays choisis par elle ont été autorisées à discuter avec elle. 